\title{
Current Design of the SSC Interaction Regions
}

\author{
Y. Nosochkov, E. Courant ${ }^{\dagger}$, A. Garren, D.M. Ritson $\ddagger$, T. Sen and R. Stiening \\ Superconducting Super Collider Laboratory * \\ Dallas, TX 75237 \\ $\dagger$ Visitor from Brookhaven Lab \\ $\ddagger$ SLAC, Stanford University, Stanford, CA 94309
}

\section{Abstract}

We review the important changes made to the optics of the Interaction Regions (IRs) in the collider since the conceptual design (SCDR) of 1990. The most significant modification is a new procedure for the $\beta$ squeeze. Three additional families of independently powered quadrupoles change the optics from injection to collision while the strengths of the final focus triplet quadrupoles are held constant. The phase advance from the interaction point (IP) to the arc quadrupoles is optimized to provide more effective positions of the local correctors for the IR. Two secondary foci where the IP is imaged have been incorporated symmetrically on both sides of the IP. Other changes include a significant reduction in the $\beta_{\text {peak }}$ at injection, optimized configuration of the $M=-I$ section, quadrupoles with $5 \mathrm{~cm}$ bore, minimized number of quad families, standard design of the vertical bends and reduced total length of the magnets. The optics allows for a wide range of values for a detector space at each IP. The time scenario for the $\beta$ squeeze is considered as well.

\section{INTRODUCTION}

The design of the IRs at the SSC has undergone several modifications since the SCDR design [1]. At present there are four IRs in the collider, two in the East cluster and two in the West. Present plans call for the detectors in the East IRs to operate at lower $\beta^{*}$ than those in the West cluster. The modifications of the IR optics are the following : - exclusion of the final triplet quadrupoles from the $\beta$ squeeze between injection and collision optics, thus providing an independent squeeze for each ring;

- including a secondary focus on either side of the IP;

- providing a specific value of the phase advance across half an IR to simplify local IR corrections;

- providing a wide range of possible values for detector space with minimal optical modifications;

- use of standard $5 \mathrm{~cm}$ bore magnets in the IR.

Here we review the above changes and discuss the optical and geometrical properties of the various IR configurations. The above modifications to the IR have not affected the rest of the machine.

\section{MAIN IR MODULES}

Figure 1 shows a vertical schematic view of a complete IR. The dipoles straddle the horizontal lines, the focussing

* Operated by the Universities Research Association Inc., for the U.S. Department of Energy, under contract DE-AC35-89ER40486

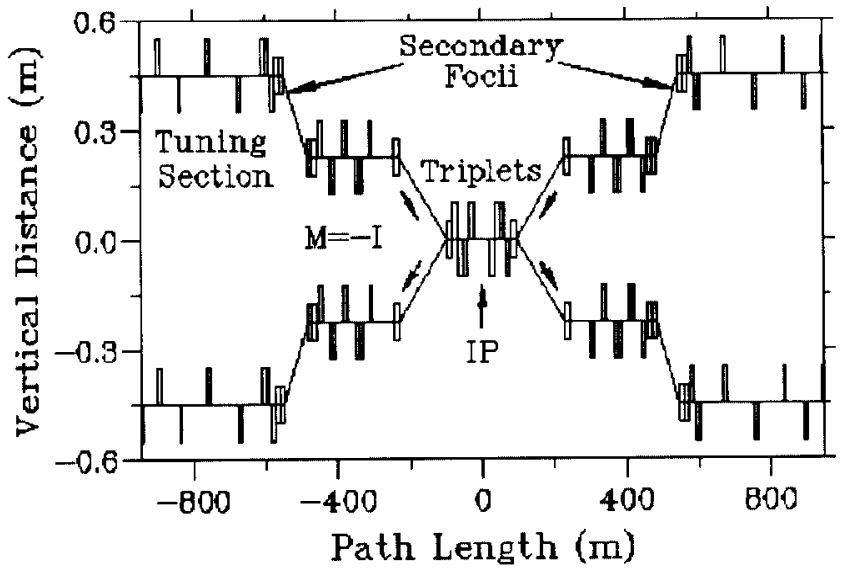

Figure 1. Vertical view of an IR.

quadrupoles are shown above the line and the defocussing quadrupoles below. As before, the optics is antisymmetric with respect to the IP. Each half IR is composed of three modules : the final triplet, the $M=-I$ section and the tuning section. Besides the quadrupoles there are vertical dipoles placed in two steps, which bring the beams into collision. The final triplet quadrupoles and adjacent splitting dipoles are common to both rings. The beams share the same beam pipe inside these magnets. There is a vertical separation of $45 \mathrm{~cm}$ between the beams in the $M=-I$ sections, which requires a 2 -in- 1 magnet design. The magnets in the tuning section in the two rings are separated by the standard vertical distance of $90 \mathrm{~cm}$.

The triplets focus the beams to extremely small sizes at the IP. Therefore, these magnets are quite strong and the beam is very sensitive to field errors in them. The most important change made in the present design is to keep these quadrupoles at fixed gradients during the $\beta$ squeeze. The advantages are that the squeeze can be done independently for each ring and no additional field errors are introduced in the triplets during the squeeze. To keep the same number of controls for the $\beta$ squeczc, another 3 families of quadrupoles, now labelled QL7, QL8, QL9, from the regions outside the previous IR were included into the IR. The 6 tuning quadrupoles QL4, QL5,.., QL9 form the tuning section. This change has increased the length of the IR from $1350 \mathrm{~m}$ to $1890 \mathrm{~m}$.

Another new feature is the inclusion of a secondary fo- 


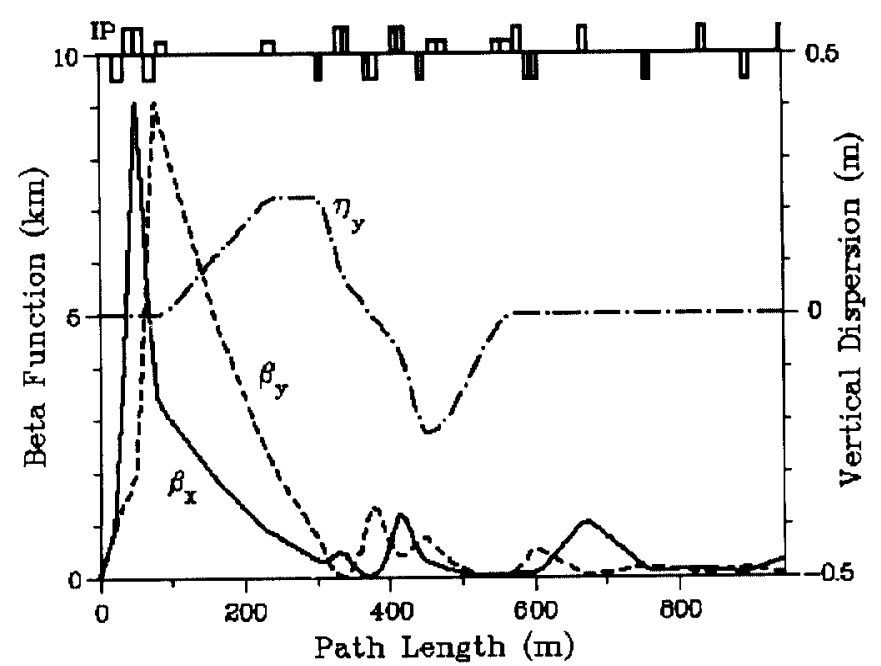

Figure 2. Lattice functions in one-half of the IR with magnets at the top.

cus, a point at which the IP is imaged. This image can be used for diagnostics, and protection collimators can be placed here to reduce the background at the IP. Two secondary foci are located at $541 \mathrm{~m}$ from the IP, symmetrically on each side of the IR, with fixed $2 \pi$ phase advance from the IP. They optically separate the two tuning sections which have variable transfer matrices from the central region (comprised of the two $M=-I$ and two triplets) with constant gradients.

Between the triplets and the tuning section is a module of 8 quadrupoles, known as the $M=-I$ section. The transfer matrix across this section is the negative of the identity matrix, hence the name. This section causes a cancellation of the vertical dispersion $\eta_{y}$, induced by the adjacent pairs of dipoles, at the IP. The length of these quadrupoles was significantly reduced by use of the FODO configuration instead of FOFDOD. The $M=-I$ section can be placed anywhere between the two sets of dipoles without affecting the rest of the IR. 'lo reduce the chromaticity at collision and provide the most detector space, this section was moved as far as possible from the IP.

\section{OPTICAL PROPERTIES}

A primary optical goal is to provide a broad range of accessible low $\beta^{*}$ values. In the present design, the variable gradient quadrupoles can be tuned to a minimum $\beta^{*}=0.25 \mathrm{~m}$ for a detector free space of $L^{*}=20.5 \mathrm{~m}$, which is defined to be the distance between the IP and the triplet. However, the baseline design requires only $\beta^{*}=0.5 \mathrm{~m}$ at collision. This corresponds to a $\beta_{\text {peak }}$ in the triplets of $9 \mathrm{~km}$. This is the maximum value allowing a region of a good field quality in the $5 \mathrm{~cm}$ bore triplet quadrupoles. Figure 2 shows the lattice functions across one-half of the IR at collision with $\beta^{*}=0.5 \mathrm{~m}$. It is envisaged that future upgrades will increase the triplet bore thus allowing $\beta^{*}=0.25 \mathrm{~m}$ to be achieved.
At injection, $\beta^{*}$ is chosen to be $7 m$ which is when the chromaticity of the IR and the $\beta_{\text {peak }}$ are a minimum. The $\beta_{\text {peak }}$ at injection is significantly reduced, from $1.3 \mathrm{~km}$ in the SCDR design to $670 \mathrm{~m}$ at present, by reversing the polarity of the doublet quadrupoles QL4, QL5.

In the earlier design the phase advance across an IR was kept constant during the squeeze so that only the sum of phase advances, $\mu_{x}+\mu_{y}$, across half an IR was held constant, but not each of the terms. Present plans include a set of local trim quadrupoles and sextupoles, correcting for the crossing angle [2] and IR chromaticity [3], located next to the quadrupoles in the arc cells adjacent to the IR. To optimize the above corrections, we require that $\mu_{x}$ and $\mu_{y}$ be separately constant during the $\beta$ squeeze. The specific values chosen are $\mu_{x}=3.75 \pi$ and $\mu_{y}=3 \pi$ across half an IR and exchanged due to antisymmetry on the other side of the IP. With $90^{\circ}$ phase advance per cell in the arcs, this makes the phase advance between the IP and the outside cell quadrupoles to be a multiple of $\pi / 2$, i.e.

$$
\mu_{x}^{F}-\mu_{x}^{I P}=n_{x} \pi / 2 ; \quad \mu_{y}^{D}-\mu_{y}^{I P}=n_{y} \pi / 2
$$

where for instance $\mu_{x}^{F}$ is the horizontal phase at an arbitrary $\mathbf{F}$ quad in the arcs. The above correctors are now at optimal positions with respect to the triplet quadrupoles, the main sources of IR perturbations. This choice also keeps the phase advance between adjacent IPs to be $\pi / 2$ ( $\bmod 2 \pi$ ) which minimizes the higher order chromaticity of the two IRs combined.

\section{MAGNET PARAMETERS}

In the present design we use optimized, realistic parameters of the IR magnets. All the quadrupoles have $5 \mathrm{~cm}$ bore and maximum gradient of $191 \mathrm{~T} / \mathrm{m}$. The quadrupole gradients in the triplets and $M=-I$ sections are not changed during the $\beta$ squeeze while the tuning quadrupoles have variable gradients. The $5 \mathrm{~cm}$ bore vertical dipoles have the same design as the horizontal dipoles in the arcs. Only the dipoles located next to the triplets need the larger aperture of $8.5 \mathrm{~cm}$ to provide enough space for the separated beams.

The positions of the quadrupoles in the tuning section and configuration of the $M=-I$ section were optimized to reduce the total length of magnets and number of different quad lcngths. Excluding the triplets, there are only 3 different lengths of IR quadrupoles : $8.0 \mathrm{~m}, 8.6 \mathrm{~m}$ and $10.2 \mathrm{~m}$. Compared to the previous IR design, the total length of magnets is reduced by $12 \%$, which is about $392 \mathrm{~m}$ for 4 IRs.

\section{BETA SQUEEZE}

The characteristic feature of the low- $\beta$ optics is an extremely high $\beta_{\text {peak }}$ value in the final triplets at collision optics. A much lower $\beta_{\text {peak }}$ value is allowed at injection energy because of the larger beam emittance. A smooth transition between the two optical configurations is achieved by 


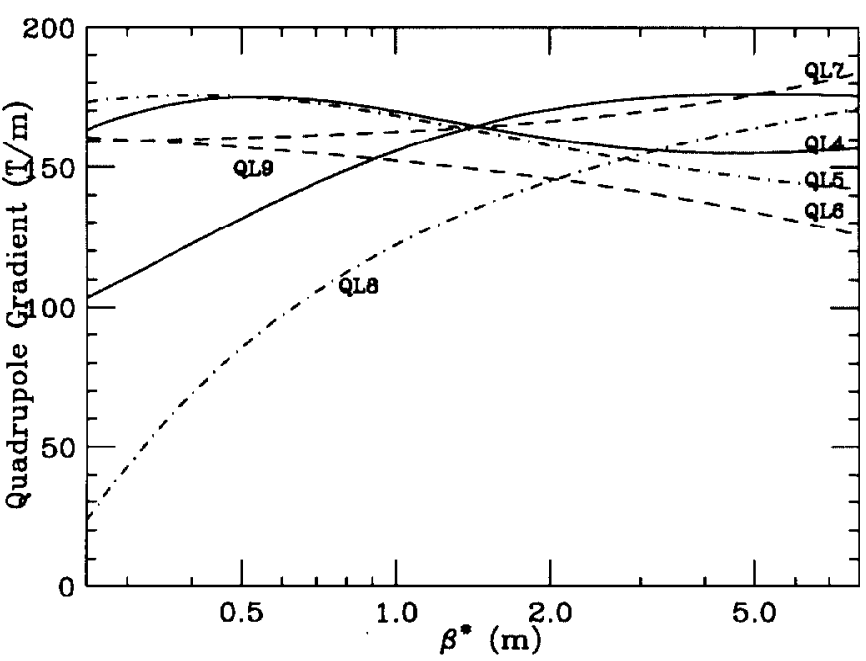

Figure 3. Tuning curves for a low- $\beta$ IR $\left(L^{*}=20.5 \mathrm{~m}\right)$.

varying the gradients of the six quadrupoles in the tuning section. This requires that each tuning quad have an independent power supply. The variation of gradients during the $\beta$ squeeze is shown in Figure 3. It is seen that only the power supply across QL8 will have a large swing from injection to collision and the other quadrupoles may use weaker power supplies. In contrast to the SCDR design, there is no reversal of polarity during the $\beta$ squeeze which makes the present design more robust.

The transfer matrix across the IR and the phase advance across each half IR stay constant during the squeeze. This guarantees that the rest of the machine is unaffected in the absence of errors.

Figure 3 shows a slow change of the gradients in the high $\beta^{*}$ part of the $\beta$ squeeze and a fast change in the low $\beta^{*}$ part. The chromaticity of the IR quadrupoles behaves similarly during the squeeze. A non-uniform time dependence of $\beta^{*}$ must be specified to make the variations more uniform in time. Minimizing both the rate of change of current in the tuning quadrupoles and the rate of change of chromaticity through the IR leads to the following exponential variation,

$$
\beta^{*}(t)=6.8 \exp (-t / 20.36)+0.2
$$

The duration of the squeeze is chosen to be 100 seconds which is the least time consistent with magnet specifications for $d I / d t$ and it is also the duration used at Fermilab. Figure 4 shows the rate of change of current through each quadrupole during the squeeze.

\section{DIFFERENT L*}

The four IRs envisaged in the collider might have different experimental requirements, such as space for the detector and beam size at the IP. Any $L^{*}$ in the range between $20.5 \mathrm{~m}$ and $90 \mathrm{~m}$ can be obtained in the present design by a simple modification. To achieve a specific value of $L^{*}$, the

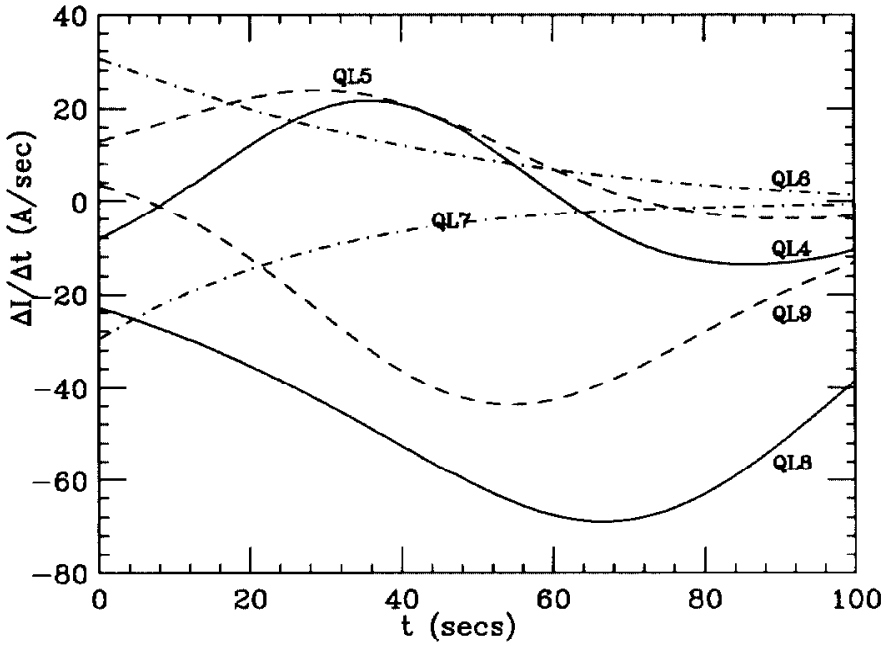

Figure 4. Rate of change of current during the squeeze $\left(L^{*}=20.5 \mathrm{~m}\right)$.

triplet and adjacent pair of the dipoles are moved together by an appropriate distance from the IP while the other magnets stay at the same positions. The lengths of the triplet quadrupoles are adjusted to keep the secondary focus at the same distance from the IP and to minimize $\beta_{\text {peak }}$ in the triplet. As $L^{*}$ increases, the triplets get shortcr bccause of the longer focal length. Slight changes are required for the gradients of the tuning quadrupoles.

For any given $L^{*}$, the lowest $\beta^{*}$ at collision corresponds to $\beta_{\text {peak }}=9 \mathrm{~km}$ in the triplet. Injection optics requires a minimum of both $\beta_{\text {peak }}$ and chromaticity over the IR. With these conditions, the range of the tuning gradients is about the same for any $L^{*}$. The following table shows the relevant parameters for the four values of $L^{*}$ chosen.

\begin{tabular}{|c||c|c||c|c|c|}
\hline \hline \multicolumn{1}{|c||}{$L^{*}(\mathrm{~m})$} & \multicolumn{3}{c||}{$\beta^{*}(\mathrm{~m})$} & \multicolumn{3}{c|}{ Triplet Quad Lengths (m) } \\
& Inj. & Coll. & QL1 & QL2 & QL3 \\
\hline 20.50 & 7.0 & 0.5 & 15.5650 & 11.8545 & 13.1715 \\
\hline 34.55 & 11.0 & 0.7 & 12.6064 & 11.1793 & 12.6064 \\
\hline 56.90 & 23.0 & 1.1 & 10.2948 & 10.2948 & 11.8835 \\
\hline 90.00 & 40.0 & 1.95 & 8.6890 & 9.4405 & 11.2051 \\
\hline \hline
\end{tabular}

The values of $34.55 \mathrm{~m}$ and $56.90 \mathrm{~m}$ were chosen since in these cases two of the quadrupoles in the triplet could be made to have the same length. The two smaller values of $L^{*}$ can be used for the low- $\beta$ IRs in the East cluster, and possibly the other two values might be used for the IRs in the West cluster.

\section{REFERENCES}

[1] J.R. Sanford, D.M. Mathews, Eds., Site-Specific Conceptual Design, SSCL Document SSCL-SR-1056 (1990).

[2] Y. Nosochkov and D. Ritson, "The Provision of IP Crossing Angles for the SSC", these proceedings.

[3] T. Sen et. al., "Chromaticity Correction for the SSC Collider Rings", these proceedings. 